\title{
Experimental studies on equivalent thermal properties of particle-reinforced flexible mould materials
}

\author{
A. K. Nandi, C. Cingi \& J. Orkas \\ Department of Engineering Design and Production, \\ Foundry Engineering, Helsinki University of Technology, Finland
}

\begin{abstract}
The investment casting process uses wax patterns which may be produced using the soft tooling process where the common flexible (polymer) mould materials used are polyurethane rubber, silicone rubber, etc of a typical kind. However, due to poor thermal conductivity of these materials, solidification time of (wax) patterns takes longer yielding to reducing rapidity of the process to a great extent. This problem may be overcome by increasing thermal conductivity of mould material that can be achieved either by molecular orientation of polymer itself or by addition of conductive fillers into polymer. The method of controlled addition of thermal conductive (particulate) fillers into mould material may be adopted by realizing it as a most simpler and easy to implement technique in industry. In this article, we have carried out an experimental study to find the affects on equivalent thermal properties of flexible mould materials reinforced with conductive filler particles. Two different types of particles (aluminium and graphite) with different morphological characteristics are considered as fillers. The measurement of thermal properties is carried out by transient plane heat source technique. It is found that as much as 10 -fold increases in thermal conductivity and thermal diffusivity values of mould materials reinforced with conductive fillers. In explaining the experimental results by several empirical/semi-empirical models, it is observed that the Lewis-Nielsen model provides a good estimation, while the Agari-Uno model (fitted with experimental data) shows better agreement than other models.

Keywords: soft tooling process, flexible mould material, particle reinforced polymer composite, equivalent thermal properties, transient plane heat source technique.
\end{abstract}




\section{Introduction}

Among different rapid tooling processes, soft tooling (ST) is the one where polymeric flexible materials (such as polyurethane (PU) rubber and silicone rubber (SR) of typical kind) are used for making mould [1]. ST process is particularly suitable to produce wax patterns (used in investment casting process) in small batch by vacuum assisted or gravity casting method based on RP (rapid prototype)/others pattern. Development of metal component through rapid prototyping-soft tooling-investment casting process is illustrated in Figure 1.

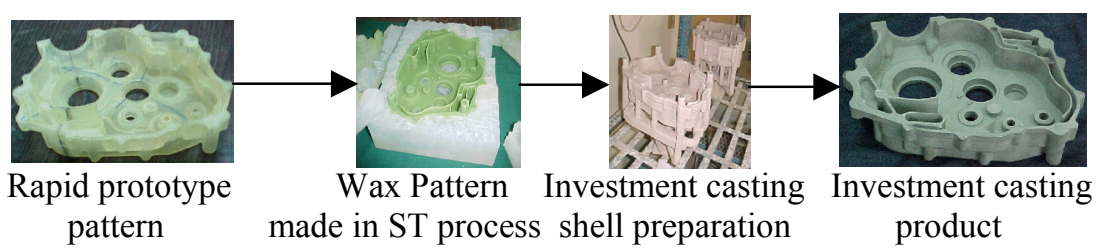

Figure 1: Development of metal components through RP-ST-wax patterninvestment casting.

However due to poor thermal conductivity (TC) of flexible mould materials, the solidification time of wax component in ST process takes long yielding to reducing the rapidity of the process to a great extent. This problem may be overcome by increasing the thermal conductivity of mould material that can be achieved either by molecular orientation of the polymer itself or by addition of conductive fillers in the polymer. The method of controlled addition of thermal conductive (particulate) fillers into mould material (to form polymer composite) is adopted by many practitioners by realizing it as a most simpler and easy to implement technique in industry. However presence of filler particles in mould material will affect other properties of mould (namely, stiffness of mould, flow ability of melt mould material, etc) those are important for ST process to obtain various advantages in moulding process. Thus, it is very much important to have a prediction on equivalent thermal conductivity of composite prior to the addition of certain amount of given filler into mould material in order to keep various advantages of ST process obtained in the mould up to the desired levels.

In past, various researchers studied the effect of equivalent thermal/electrical conductivity of polymer by inclusion of metallic/ceramic powders. A significant amount of work has been conducted by varying the amount of conductive fillers in composite materials [2-5]. The aim of present research work is to analyse the effect on equivalent thermal properties (thermal conductivity, thermal diffusivity and specific heat) of flexible mould materials, PU and SR by addition of high thermal conductive filler particles, aluminium and graphite. In addition, various equivalent thermal conductive models reported in literature are used to explain the experimental results of thermal conductivities. 


\section{Materials and composite manufacturing}

\subsection{Flexible mould materials}

Among different mould materials, in this study we have chosen two flexible mould materials extensively used in industries, namely polyurethane (PU) produced by Smooth-ON, Inc. USA and silicone rubber (SR) (Silicones ELASTOSIL $^{\circledR}$ RT 601 A) manufactured by Wacker-Chemie GMBH, Germany. Both PU and SR are of two parts (part A and part B) mixing in the ratio of 1:1 and 9:1 by weight or volume, respectively and cured in room temperature.

\subsection{Filler particle}

The filler materials that we have considered in this work are aluminium fine powder and graphite granules having thermal conductivities 237 and 209.34 $\mathrm{W} / \mathrm{m}-\mathrm{k}$, respectively. The average filler particle size and shape distributions are investigated through experimentation as follows:

Table 1: $\quad$ Filler particle size and shape distributions.

\begin{tabular}{|c|c|c|c|c|c|c|c|}
\hline Filler & \multicolumn{4}{|c|}{ Size distributions } & \multicolumn{3}{|c|}{ Shape distributions } \\
\hline Graphite & $\begin{array}{c}\text { From } \\
0.1 \mathrm{~mm} \\
\text { to } \\
0.3 \mathrm{~mm}\end{array}$ & $\begin{array}{c}\text { Above } \\
0.3 \mathrm{~mm} \\
\text { to } \\
0.5 \mathrm{~mm}\end{array}$ & $\begin{array}{c}\text { Above } \\
0.5 \mathrm{~mm} \\
\text { to } \\
1.0 \mathrm{~mm}\end{array}$ & $\begin{array}{l}\text { Above } \\
1.0 \mathrm{~mm}\end{array}$ & Spherical & Cylindrical & Irregular \\
\hline & $72 \%$ & $18 \%$ & $8 \%$ & $2 \%$ & $30 \%$ & $27 \%$ & $43 \%$ \\
\hline$\underset{\mathrm{m}}{\text { Aluminiu }}$ & $\begin{array}{l}\text { From } \\
1 \mu \mathrm{m} \text { to } \\
10 \mu \mathrm{m}\end{array}$ & $\begin{array}{l}\text { Above } \\
10 \mu \mathrm{m} \text { to } \\
30 \mu \mathrm{m}\end{array}$ & $\begin{array}{c}\text { Above } \\
30 \mu \mathrm{m} \text { to } \\
50 \mu \mathrm{m}\end{array}$ & $\begin{array}{l}\text { Above } \\
50 \mu \mathrm{m}\end{array}$ & Spherical & Cylindrical & Irregular \\
\hline & $66 \%$ & $24 \%$ & $8 \%$ & $2 \%$ & $46 \%$ & $22 \%$ & $32 \%$ \\
\hline
\end{tabular}

\subsubsection{Filler particle size and shape distributions}

Measurements of granulometric parameters of both the fillers are made using M420, WILD Heerbrugg Microscope. The particle size and shape distributions of both filler materials are enlisted in Table 1. The (arithmetic mean) size of particles is determined by taking the average of minimum and maximum lengths of the particles. For (near) spherical shape, the particle size is equivalent to the diameter of sphere. For (near) cylindrical shape, the size of particle is equal to (length + diameter) $/ 2$. In order to find the size distribution of particles, a small amount of sample is taken at random form bulk. We have counted the number of particles in that amount as well measured the size of each particle in an approximate approach and finally determine the particle size distributions arithmetically in four different ranges. The average particle sizes (calculated based on weighted average method) of aluminium and graphite fillers are 12.3 micron and 296 micron, respectively. The maximum volume fractions of graphite and aluminium particles found experimentally are 0.5216 and 0.6032 , respectively. 


\subsection{Composite manufacturing}

In order to prepare the composite samples (50 mm square and thickness 5-6 mm) used for thermal property measurements, the weights of part-A and part-B of liquid polymer (PU/SR) and filler are calculated based on the densities of these components for a certain volume fraction of filler in the composite. Then the part A (liquid polymer) is first mixed with filler particles in a container using manual stirring. To assure a uniform mixing, stirring of liquid polymer and gradual pouring of filler material is done simultaneously. Once, the uniform mixing of liquid polymer and filler material is achieved, the hardener (Part B) will be pored into the mixture of liquid polymer and filler, and stirred it properly for another 5-10 minutes. Before pouring the mixture material into mould box, release agent is sprayed thoroughly inside the mould box. After curing the polymer composite at room temperature, it is taken out from mould box and the mould box is reused for preparing another sample. Diffe rent samples are prepared by varying the amount of (volume fraction) filler mixed with PU and SR. We have restricted the amount of filler to be mixed with mould material up to the maximum packing fraction of filler particles since it indicates the maximum loading level of particles to be mixed into a fluid.

\section{Measurement of thermal properties}

Thermal properties of different composites are measured based on transient plane heat source (TPS) hot disk method [6] following the standard, ISO 22007-2: 2008(E). We have used the instrument, TPS 2500 S Thermal Conductivity System associated with the software, HotDisk Thermal Constant Analyser V.5.9.5 (solids, liquids, powders and paste) [7-9]. The TPS $2500 \mathrm{~S}$ Thermal Conductivity System utilizes a hot disk sensor in the shape of a double spiral of nickel wire. The hot disk sensor (design number: C5501) with 14 concentric rings is used here for measuring thermal properties. The sensor element is made of $10 \mu \mathrm{m}$ thick nickel wire and the spiral is supported by $30 \mu \mathrm{m}$ thick Kapton material to protect its particular shape by providing mechanical strength and also keeps it electrically insulated. The relaxation time of the probe is less than $10 \mathrm{~ms}$ and the required time to reach a constant temperature difference is kept as $15 \mathrm{~min}$. The sensor used in the experiments has a diameter of $2 \mathrm{a}$ ( $\mathrm{a}$ is the radius of largest ring, $6.403 \mathrm{~mm}$ ) and total thickness (Kapton-nickel-Kapton) of $2 \mathrm{v}(=70 \mu \mathrm{m})$ was placed between two samples of $50 \mathrm{~mm}$ square and thickness $5-6 \mathrm{~mm}$. The probing depth of the sensor used for all the samples is around $6 \mathrm{~mm}$. In hot disk method, measurements of thermal properties are made based on the average temperature increase in hot disk sensor. In the following, derivation of average temperature increase in hot disk sensor and the method of computing thermal properties are made.

The differential equation of heat conduction in an isotropic material whose thermal conductivity is independent of temperature is given by [10]

$$
k \nabla^{2} T+\frac{Q}{\rho c}=\frac{\partial T}{\partial t}
$$


where $\mathrm{k}(=K / \rho c)$ is the thermal diffusivity, $K$ is the thermal conductivity, $\rho$ and $c$ are the density and specific heat of material, respectively. $\rho c$ is called volumetric specific heat of the material. $T(x, y, z, t)$ is the temperature at point $(\mathrm{x}, \mathrm{y}, \mathrm{z})$ and time, $t$. $Q(x, y, z, t)(\mathrm{J} / \mathrm{m} 3-\mathrm{s})$ is the amount of heat released at $(\mathrm{x}, \mathrm{y}, \mathrm{z}, \mathrm{t})$ per unit volume and unit time.

The fundamental solution of Equation (1), assuming that heat source switched at time $t=0$, is

$$
T(\vec{r}, t)=T_{0}+\frac{1}{\rho c\left(4 \prod k\right)^{3 / 2}} \int_{0 V^{\prime}}^{t} \int \frac{Q\left(\vec{\xi}, t^{\prime}\right)}{\left[\left(t-t^{\prime}\right)\right]^{3 / 2}} e^{\left(-\frac{(\vec{r}-\vec{\xi})^{2}}{4 k\left(t-t^{\prime}\right)}\right)} d^{3 \vec{\xi}} d t^{\prime}
$$

In cylindrical coordinates, any position in the sample is coordinates, any position in the sample is $\vec{r}=(r, \theta, z)$, any position in the source is $\vec{\xi}=\left(r^{\prime}, \theta^{\prime}, z^{\prime}\right)$ and $(\vec{r}-\vec{\xi})^{2}=r^{2}+r^{\prime 2}-2 r r^{\prime} \cos \left(\theta-\theta^{\prime}\right)+\left(z-z^{\prime}\right)^{2}$.

For continuous single ring source with radius, a in the $z^{\prime}=0$ plane, the heat source strength can be expressed as

$$
Q=Q_{0} \delta\left(r^{\prime}-a\right) \delta\left(z^{\prime}\right) u\left(t^{\prime}\right)
$$

in which $\delta()$ is the Dirac delta function (where $\int_{-\alpha}^{+\alpha} f(x) \delta(x-p)=f(p)$ ) and $u\left(t^{\prime}\right)$ (where $u\left(t^{\prime}\right)=0$ for $t^{\prime}<0$ and $u\left(t^{\prime}\right)=1$ for $t^{\prime} \geq 0$ ) is the Heaviside unit step function. $Q_{0}$ is the heat released per unit length of ring source.

A hot disk sensor is considered to have m number of concentric rings (heat) source those are equally spaced [6]. Assuming the source is continuous and it is switched on at $t^{\prime}=0$, the strength of heat source for can be defined as

$$
Q=Q_{0} \sum_{l=i}^{m} \delta\left(r^{\prime}-\frac{l a}{m}\right) \delta\left(z^{\prime}\right) u\left(t^{\prime}\right)
$$

where $a$ is the radius of the largest ring and that of the smallest ring is $\frac{a}{m}$, respectively and the total length (heating filament) ring $(L)$ is $\sum_{n=1}^{m} 2 \prod \frac{n a}{m}$ (i.e., $\left.(m+1) \prod a\right)$. Thus, the total heat released by the sensor in time $\mathrm{t}$ is obtained as

$$
\begin{gathered}
H=\iint_{V^{\prime}} \int_{0}^{t} Q\left(\xi^{\prime}, t^{\prime}\right) d V^{\prime} d t \\
=\int_{0}^{\alpha} Q_{0} \sum_{l=1}^{m} \delta\left(r^{\prime}-\frac{l a}{m}\right) \delta\left(z^{\prime}\right) r^{\prime} d r^{\prime} \int_{0}^{2 \prod} d \theta^{\prime} \int_{-\alpha}^{+\alpha} d z^{\prime} \int_{0}^{t} u\left(t^{\prime}\right) d t^{\prime}
\end{gathered}
$$


370 High Performance Structures and Materials V

$$
\begin{gathered}
=\prod a(m+1) Q_{0} t \\
=P_{0} t
\end{gathered}
$$

where $P_{0}$ is the power output of the hot disk sensor per unit time. Now, the temperature increase caused by hot disk sensor can be obtained by carrying out the integration in Equation (2) and with the help of Equation (4),

$$
\begin{aligned}
& T(\vec{r}, t)-T_{0}=\frac{\mathcal{Q}_{0}}{\rho c} \sum_{l=1}^{m} \int_{0}^{\alpha} \delta\left(r^{\prime}-\frac{l a}{m}\right) e^{\frac{-\left[r^{2}+r^{\left.\prime^{2}-2 r r^{\prime} \cos \left(\theta-\vartheta^{\prime}\right)\right]}\right.}{4 k\left(t-t^{\prime}\right)}} r^{\prime} d r^{\prime}
\end{aligned}
$$

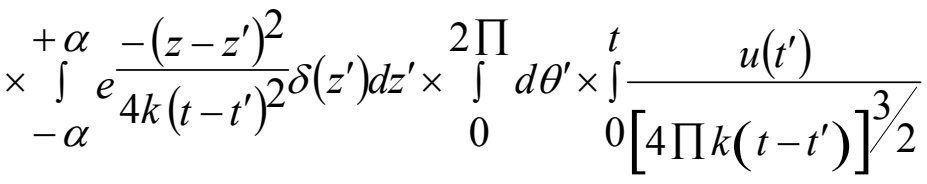

$$
\begin{aligned}
& =\frac{Q_{0}}{\rho c} \sum_{l=1}^{m} \frac{l a}{m} e^{\frac{-\left(r^{2}+\left(\frac{l a}{m}\right)^{2}+z^{2}\right)}{4 k\left(t-t^{\prime}\right)}} \int_{0}^{t} \frac{d t^{\prime}}{\left[4 \prod k\left(t-t^{\prime}\right)\right]^{3 / 2}} \int_{0}^{2 \prod} e^{\frac{r l a}{2 m k\left(t-t^{\prime}\right)} \cos \left(\theta-\theta^{\prime}\right)} d \theta^{\prime} \\
& =\frac{2 \prod a Q_{0}}{\rho c m} \sum_{l=1}^{m} l e^{-\left(r^{2}+\left(\frac{l a}{m}\right)^{2}+z^{2}\right)} \int_{0}^{t k\left(t-t^{\prime}\right)} \frac{d t^{\prime}}{\left[4 \prod k\left(t-t^{\prime}\right)\right]^{3 / 2}} I_{0}\left(\frac{r l a}{2 m k\left(t-t^{\prime}\right)}\right)
\end{aligned}
$$

where $I_{0}(x)=\frac{1}{2 \prod} \int_{0}^{2 \Pi} e^{x \cos \theta} d \theta=\frac{1}{2 \prod} \int_{0}^{2 \Pi} e^{x \sin \theta} d \theta$ is the first kind modified Bessel function of the $0^{\text {th }}$ order.

In a hot a hot disk measurement as the temperature change near the surface of the sensor is concerned, $z \rightarrow 0$ and by considering the power output of the hot disk sensor per unit time $\left(P_{0}\right)$ instead of $Q_{0}$, Equation (7) may be written as

$$
\Delta T(r, t)=\frac{P_{0}}{\rho c m(m+1)} \int_{0}^{t} \frac{d t^{\prime}}{4\left[\prod k\left(t-t^{\prime}\right)\right]^{3 / 2}} \sum_{l=1}^{m} l e^{\frac{-\left(r^{2}+\left(\frac{l a}{m}\right)^{2}\right)}{4 k\left(t-t^{\prime}\right)}} I_{0}\left(\frac{r l a}{2 m k\left(t-t^{\prime}\right)}\right)
$$

Equation (8) may be rewritten by introducing some new parameters as follows

$$
\Delta T(r, \tau)=\frac{P_{0}}{2 \prod^{3 / 2} a m(m+1) k \rho c} \int_{0}^{\tau} \frac{d \sigma}{\sigma^{2}} \sum_{l=1}^{m} l e^{\frac{\left.-(r / a)^{2}+(l / m)^{2}\right)}{{ }^{4} \sigma^{2}}} I_{0}\left(\frac{r l}{2 m a \sigma^{2}}\right)
$$


where $\sigma$ is an integration variable that defined as $\sqrt{\frac{k\left(t-t^{\prime}\right)}{a^{2}}}$ and the (dimensionless) parameter, $\tau\left(=\frac{\sqrt{k t}}{a}\right)$ is called the characteristic time ratio.

Thus the temperature increase at any point on the sensor surface (i.e., $\mathrm{z}=$ plane), is described by the Equation (9). But to determine the temperature increase of the sensor itself, it is required to determine the average temperature increase over the length of the concentric rings. Therefore, the average temperature increase of the sensor is defined as

$$
\Delta \bar{T}(\tau)=\frac{1}{L} \int_{0}^{2 \prod} \Delta T(r, \tau) \sum_{n=1}^{m} \delta\left(r-\frac{n a}{m}\right) r d \theta
$$

By replacing the expressions of $\Delta T(r, \tau)$ (from Equation (9)) and $L$ (as cited above) in Equation (10), $\Delta \bar{T}(\tau)$ may be expressed as

$$
\begin{aligned}
& \Delta \bar{T}(\tau)=\frac{1}{\prod a(m+1)} \times \frac{P_{0}}{2 \prod^{3 / 2} a m(m+1) K} \int_{0}^{\tau} \frac{d \sigma}{\sigma^{2}} \sum_{n=1}^{m} \frac{n a}{m} \sum_{l=1}^{m} l e^{\frac{-\left((r / a)^{2}+(l / m)^{2}\right)}{{ }^{4} \sigma^{2}}} I_{0}\left(\frac{n l}{2 m^{2} \sigma^{2}}\right) 2 \Pi \\
& =\frac{P_{0}}{\prod^{3 / 2} a K} \times \frac{1}{m^{2}(m+1)^{2}} \times \int_{0}^{\tau} \frac{d \sigma}{\sigma^{2} \sum_{n=1}^{m} n \sum_{l=1}^{m} l} e^{\frac{-\left((r / a)^{2}+(l / m)^{2}\right)}{{ }^{4} \sigma^{2}}} I_{0}\left(\frac{n l}{2 m^{2} \sigma^{2}}\right) \\
& =\frac{P_{0}}{\prod^{3 / 2} a K} D(\tau)
\end{aligned}
$$

where $K$ is thermal conductivity of materials. $D(\tau)$ is a dimensionless time function. A more detailed derivation of average temperature increase in hot disk sensor can be found in [11].

From Equation (11), it is seen that average temperature increase in hot disk sensor, $\Delta \bar{T}(\tau)$ is linearly proportional with the dimensionless time function, $D(\tau)$ and exhibited straight-line curve by plotting there values. The slope of this straight-line curve is equal to $P_{0}$ from which the value of $K$ may $\prod^{3 / 2} a K$

be evaluated. But, the straight line curve between $\Delta \bar{T}(\tau)$ and $D(\tau)$ may be obtained for a proper value of $\tau$ that is again depended on the value of $k$ (for given a given value of $a$ and time, $\mathrm{t}$ ). Therefore, it is required to find the proper value of $k$ and normally this may be done by making a series of computational plots of $\Delta \bar{T}(\tau)$ verses $D(\tau)$ for a range of $\mathrm{k}$ values. The correct value of $k$ will be reached once the straight-line plot of $\Delta \bar{T}(\tau)$ verses $D(\tau)$ is obtained. Once 
the correct value of $k$ is obtained, the value of $K$ can be determined from the slope of the straight-line plot of $\Delta \bar{T}(\tau)$ verses $D(\tau)$.

Another way to determine the value of $K$ is to measure the density $(\rho)$ and the specific heat $(c)$ of the material separately. Then, the value of $K$ can be obtained by multiplying the value of $k$ with the density and specific heat of the material. This method is generally applied for anisotropic materials. If the properties along $\mathrm{x}$ - and $\mathrm{y}$ - axes are the same, but different from those along the z-axis and if the plane of the hot disk sensor is mapped out by $x$ - and $y$ - axes, the Equation (11) will be expressed as

$$
\Delta \bar{T}\left(\tau_{x}\right)=\frac{P_{0}}{\prod^{3 / 2} a \sqrt{K_{x} K_{z}}} D\left(\tau_{x}\right)
$$

where $K_{x}$ and $K_{z}$ are the thermal conductivities in the $\mathrm{x}$ (or y) and z directions respectively and $\tau_{x}=\frac{\sqrt{k_{x}^{t}}}{a}$. The value of $k_{x}$ can be determined by the above stated iteration process until a straight-line plot of $\Delta \bar{T}\left(\tau_{x}\right)$ verses $D\left(\tau_{x}\right)$ is reached and the value $K_{x}$ is equivalent to $k \rho c$. On the other hand, from the slope of the straight-line plot of $\Delta \bar{T}\left(\tau_{x}\right)$ verses $D\left(\tau_{x}\right)$, the value of $\sqrt{K_{x} K_{z}}$ can be found out. Therefore, the value of $K_{z}$ is calculated by dividing the value of $\sqrt{K_{x} K_{z}}$ by obtained value of $K_{x}$.

\section{Results and discussion}

The equivalent thermal conductivity (ETC), thermal diffusivities and volumetric heat capacity of particulate filled PU and SR composites with $\mathrm{Al}$ and graphite particles for different amount of filling fractions as obtained through experimentations at room temperature $\left(23^{\circ} \mathrm{C}\right)$ are illustrated in Figure 2. From the experimental results, it is quite evident that thermal conductivities of particulate filled PU and SR are increasing (around 10 times) with increasing amount of filler and the increasing rate starts drastically more at around $20-30 \%$ volume fraction of filler for some composites. This is due to the formation of thermal conductive chain in composite whose tendency is high in elevated filler content. In Figure 2, another point is noticed that increasing rate of thermal conductivity is comparatively higher for any level of filler content when graphite filler is used for both the mould materials. The reason is, large particles which is equivalent as the composed of aggregates of filler particles are much capable of forming conductive chains than fine particles. Moreover, the amount of heat scattered around the contact points in case of coarse particles is smaller than fine particles, since less number of contact points required to form the same length of conductive chain [2]. 


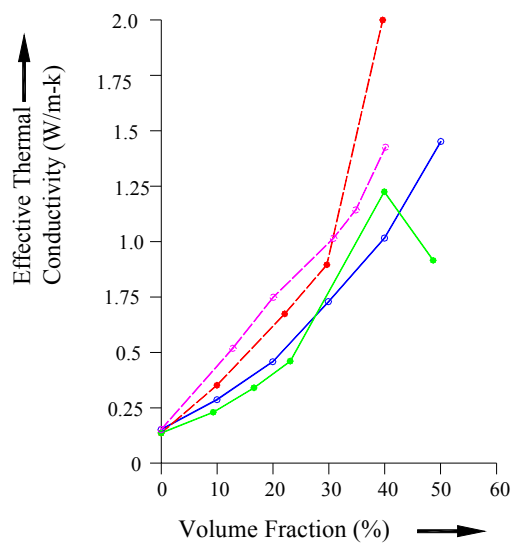

(a)

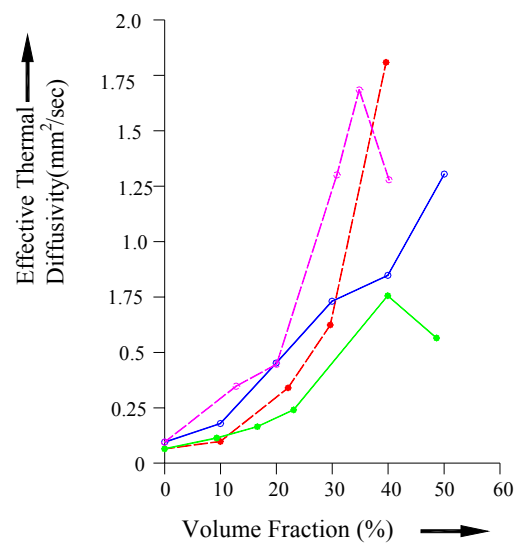

(b)

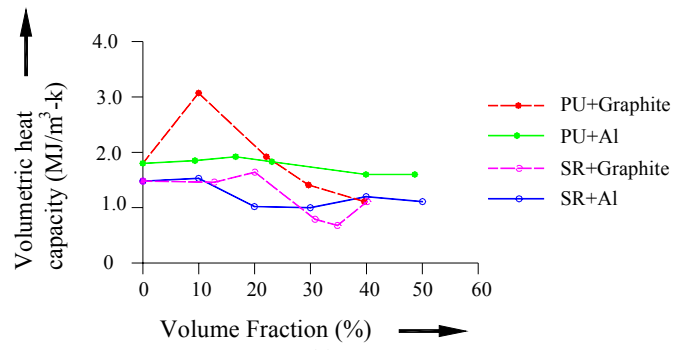

(c)

Figure 2: Equivalent thermal properties of PU and SR composites with aluminium and graphite particles: (a) thermal conductivity (b) thermal diffusivity (c) volumetric heat capacity.

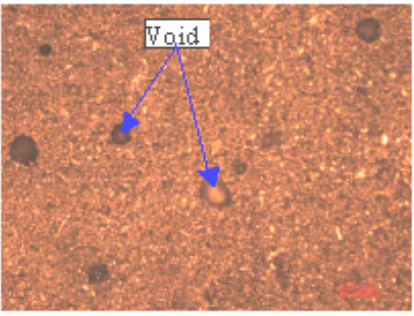

(a)

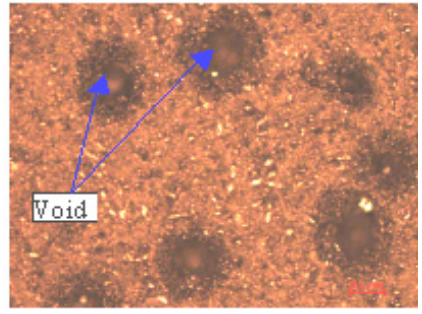

(b)

Figure 3: Morphological structure of PU and aluminium composites with volume fraction levels: a) $39.922 \%$ b) $48.664 \%$.

On the other hand, it is worth mentioning that manufacturing process of composite is an important aspect to achieve a maximum value of ETC of flexible mould material composites. Because there is much possibility of the presence of voids in mould due to fault(s) in manufacturing, which will reduce effective thermal conductivity. This phenomenon is observed in the results of PU-Al 


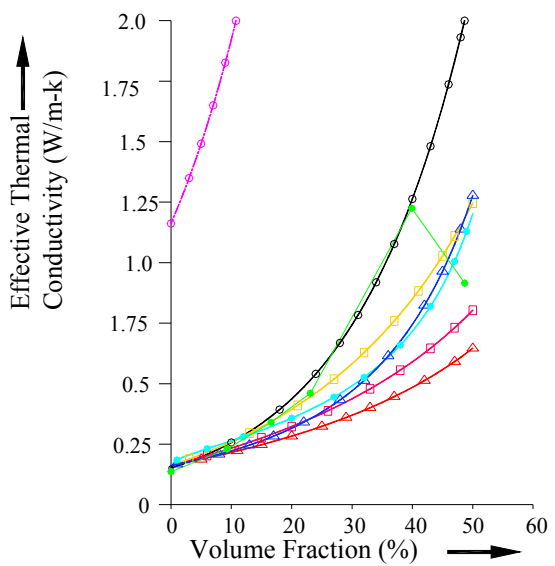

(a)

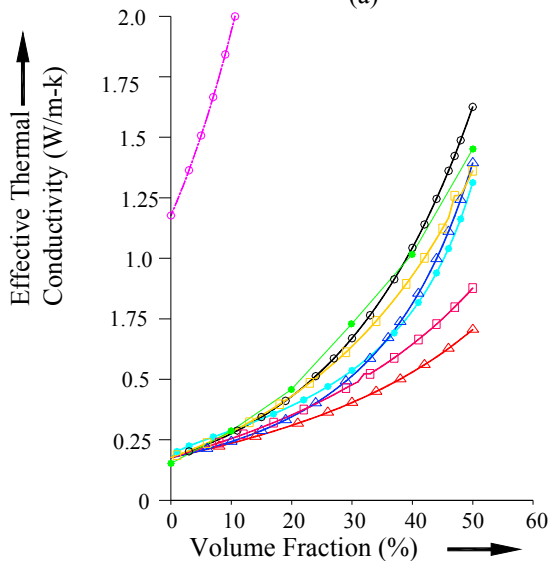

(c)

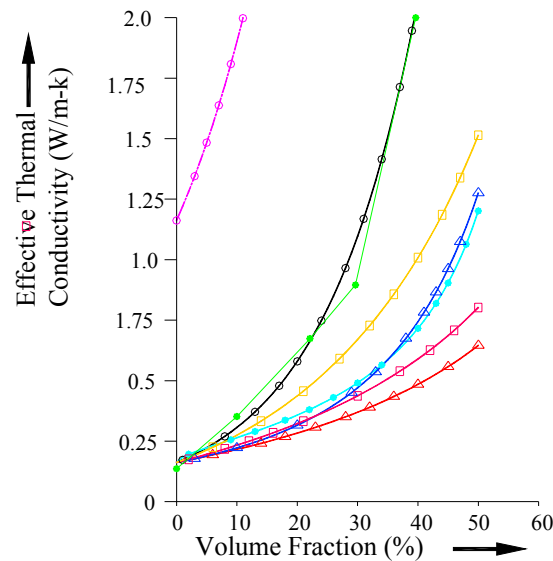

(b)

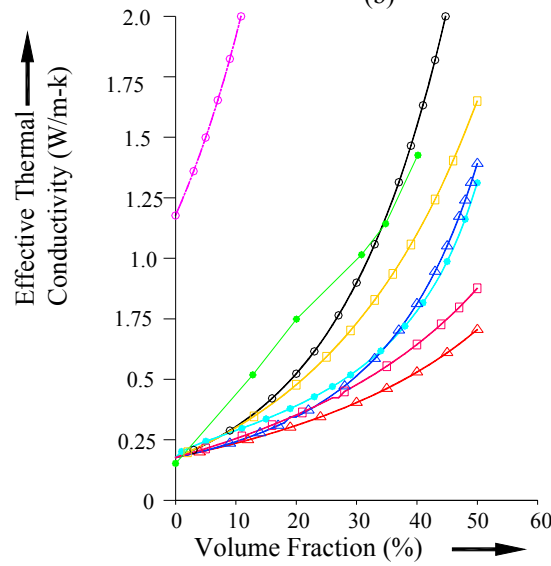

(d)

$\therefore$ Experimental $\stackrel{\triangle}{\triangle}$ Maxwell-Eucken [12] $\rightarrow$ Cheng-Vackon [13] $\rightarrow$ Ziebland [14]

$\triangle \wedge$ Bruggeman [15] $\square$ Lewis-Nielsen [16] $\square--\square$ Torquato [17] $\multimap$ Agari-Uno [18]

Figure 4: Thermal conductivities of composites (a) PU-Al (b) PU-Graphite (c) SR-Al (d) SR-Graphite: experimental values and various models.

composites (Figure 2(a)). It is found that though the composite contains higher filler (48.66\%), it exhibits lower ETC than that having lower (39.922\%) filler due to presence of more voids (as seen in Figure 3).

The experimental results of ETC of 4 kinds of composites were tried to explain by several empirical/semi-empirical models as shown in Figure 4 . It is observed that the Lewis-Nielsen model provides closer estimations (average \% error, 28.48) than others empirical models in all four composites. Moreover, a better fit is observed with the semi-empirical model of Agari-Uno whose controlling parameters are determined based on the experimental data. The average \% errors of Agari-Uno model are found as 2.35, 10.12, 8.9 and 16.82 by 
individually fitting the composite systems mentioned in Figures 4(a), 4(b), 4(c) and 4(d), respectively. However, it was found that the average \% error of the Agari-Uno model by fitting all the data of 4 composite systems is considerably high (19.29\%) though still lower than Lewis-Nielsen model. Therefore, it may be argued that development of a generic model for ETC is a difficult task and it may be achieved by use of suitable data-driven modelling technique.

In looking at the Agari-Uno models [18], it was found that values of $\mathrm{C} 1$ $(0.933594,1.0,1.0$ and 1.0$)$ are nearer to 1.0 and the values $C 2$ are 0.625 , $0.852295,0.4944$ and 0.690013 for the suspension systems, Figures 4(a), 4(b), 4(c) and 4(d), respectively. Value of $\mathrm{C} 1$ close to 1.0 indicates that effect of crystallinity and crystal size of polymer and changing thermal conductivity of polymers is negligible due to the inclusion of both graphite and Al particles. The value of $\mathrm{C} 2$ close to 1 indicates more ease in forming conductive chains of particles in composite. In the present composites, it is observed that the value of $\mathrm{C} 2$ is greater for large particle size filler (graphite) than for the smaller one (Al) which agrees well with the results Boudenne et. al [19].

\section{Conclusions}

In the present work, an experimental study is carried out to find the equivalent thermal properties of particle reinforced flexible mould materials using hot disk technique. The measurements of thermal properties using hot disk technique are presented. A significant increase of thermal conductivity of composite mould materials (PU and SR) was observed which will be helpful to reduce the cooling time in ST process. However, presence of voids in the composite due to manufacturing fault(s) reduces the effective thermal conductivity considerably. It was observed that data driven model, Agari-Uno and numerical model LewisNielsen provide better TC estimations than other models.

\section{Acknowledgements}

The authors are thankful to DST (Department of Science and Technology), New Delhi, India for supporting this research work under the BOYSCAST fellowship programme.

\section{References}

[1] Rosochowski, A. \& Matuszak, A., Rapid tooling: the state of the art. Journal of Materials Processing Technology, 106(1-3), pp. 191-198, 2000.

[2] Agari, Y. \& Uno, T., Thermal conductivity of polymer filled with carbon materials: Effect of conductive particle chains on thermal conductivity. Journal of Applied Polymer Science, 30, pp. 2225-2235, 1985.

[3] Bigg, D.M., Conductive polymeric compositions. Polymer Engineering and Science, 17(12), pp. 842-847, 1977. 
[4] Nagata, K., Iwabuki, H. \& Nigo, H., Effect of particle size of graphites on electrical conductivity of graphite/polymer composites. Composite Interfaces, 6(5), pp. 483-495, 1999.

[5] Murthy, M.V., Permanent EMI shielding of plastics using copper fibers. Proc. of the Society of Plastics Engineers Annual Technical Conference, San Francisco, New York, pp. 1396-1400, 1994.

[6] Gustafsson, S.E., Transient plane source techniques for thermal conductivity and thermal diffusivity measures of solid materials. Review of Scientific Instruments, 62(3), pp. 797-804, 1991.

[7] Gustavsson, M., Karawacki, E. \& Gustafsson, S.E., Thermal conductivity, thermal diffusivity, and specific heat of thin samples from transient measurements with hot disk sensors. Review of Scientific Instruments, 65(12), pp. 3856-3859, 1994.

[8] Log T. \& Gustafsson, S.E., Transient plane source (TPS) technique for measuring thermal transport properties of building materials. Fire and Materials, 19(1), pp. 43-49, 1995.

[9] Bohac, V., Gustavsson, M.K., Kubicar, L. \& Gustafsson, S.E., Parameter estimations for measurements of thermal transport properties with the hot disk thermal constants analyzer. Review of Scientific Instruments, 71(6), pp. 2452-2455, 2000.

[10] Carslaw, H.S. \& Jaeger, J.C., Conduction of heats in solids, 2nd ed., Oxford Science Publications, New York, 2000.

[11] He, Y., Rapid thermal conductivity measurement with a hot disk sensor Part 1. Theoretical considerations. Termochimica Acta, 436, pp. 122-129, 2005.

[12] Maxwell, J.C, A Treatise on Electricity and Magnetism, 3rd edition, Chapter 9, Dover Inc., New York, NY, 1954.

[13] Cheng, S.C. \& Vachon, R.I., The prediction of the thermal conductivity of two and three phase solid heterogeneous mixture. International journal of Heat Mass Transfer, 12(3), pp. 249-264, 1969.

[14] Butta E. \& Migliaresi, C., Materiali compositi a matrice polimerica, AIMAT Manuale dei Materiali per l'Ingegneria. McGraw Hill, ISBN883863211-1, 1996.

[15] Bruggeman, D.A.G., Berechnung Verschiedener physikalischer konstanten von heterogenen substanzen. Annals Physics (Leipzig), 24(5), pp. 636-679, 1935.

[16] Lewis T. \& Nielsen, L.E., Dynamic mechanical properties of particulatefilled composites. Journal of Applied Polymer Science, 14, pp. 1449-1471, 1970.

[17] Torquato, S., Effective electrical conductivity of two-phase disordered composite media. J Applied Physics, 58(10), pp. 3790-3797, 1985.

[18] Agari Y. \& and Uno, T., Estimation on thermal conductivities of filled polymers. Journal of Applied Polymer Science, 32(7), pp. 5705-5712, 1986.

[19] Boudenne A., Ibos L., Fois, M., Gehin, E., and Majeste, J.C., Thermophysical properties of polypropylene/aluminium composites, Journal of Polymer Science: Part B: Polymer physics, 42(4), pp. 722-732, 2004. 